Article

\title{
Development of an In Vitro System to Study the Interactions of Aerosolized Drugs with Pulmonary Mucus
}

\author{
Safar Alqahtani ${ }^{+}(\mathbb{D}$, Clive J. Roberts, Snjezana Stolnik and Cynthia Bosquillon * (B) \\ School of Pharmacy, University of Nottingham, Nottingham NG7 2RD, UK; safar.alqahtani@psau.edu.sa (S.A.); \\ clive.roberts@nottingham.ac.uk (C.J.R.); snow.stolnik@nottingham.ac.uk (S.S.) \\ * Correspondence: cynthia.bosquillon@nottingham.ac.uk; Tel.: +44-115-846-6078 \\ + Current affiliation: Pharmaceutical Chemistry Department, College of Pharmacy, Prince Sattam bin Abdul \\ Aziz University, Al-Kharj 11942, Saudi Arabia
}

Received: 11 December 2019; Accepted: 8 February 2020; Published: 11 February 2020

\begin{abstract}
Mucus is the first biological component inhaled drugs encounter on their journey towards their pharmacological target in the upper airways. Yet, how mucus may influence drug disposition and efficacy in the lungs has been essentially overlooked. In this study, a simple in vitro system was developed to investigate the factors promoting drug interactions with airway mucus in physiologically relevant conditions. Thin layers of porcine tracheal mucus were prepared in Transwell ${ }^{\circledR}$ inserts and initially, the diffusion of various fluorescent dyes across those layers was monitored over time. A deposition system featuring a MicroSprayer ${ }^{\circledR}$ aerosolizer was optimized to reproducibly deliver liquid aerosols to multiple air-facing layers and then exploited to compare the impact of airway mucus on the transport of inhaled bronchodilators. Both the dyes and drugs tested were distinctly hindered by mucus with high $\log \mathrm{P}$ compounds being the most affected. The diffusion rate of the bronchodilators across the layers was in the order: ipratropium $\approx$ glycopyronnium $>$ formoterol $>$ salbutamol > indacaterol, suggesting hydrophobicity plays an important role in their binding to mucus but is not the unique parameter involved. Testing of larger series of compounds would nevertheless be necessary to better understand the interactions of inhaled drugs with airway mucus.
\end{abstract}

Keywords: pulmonary delivery; aerosols; mucus; absorption; in vitro models

\section{Introduction}

Development efforts in pulmonary drug delivery have primarily focused on advancing device technologies and formulations in order to improve aerosol deposition in the respiratory system as well as patients' compliance [1]. Despite progresses in this field, no superior treatment of respiratory diseases by the inhaled route has been achieved in recent years and the attrition rate of inhaled drug candidates remains extremely high as compared to compounds developed for other therapeutic areas [2]. One of the principal underlying causes is a lack of understanding of the factors affecting drug disposition post-deposition in the lungs. Notably, very little is currently known on the interactions of inhaled drugs with the respiratory mucosa.

In the upper airways, the epithelium is covered by a $\sim 10 \mu \mathrm{m}$ thick mucus layer that aerosolized drugs must be able to traverse for exerting their pharmacological action as well as avoiding rapid clearance by the mucocilliary escalator. Mucus has been demonstrated to act as a permeability barrier for a range of drugs, particularly lipophilic ones, and is thus deemed to affect their efficacy [3,4]. Due to the popularity of the oral route of administration, investigations on mucus-drug interactions have nevertheless almost exclusively been performed using gastro-intestinal mucus [4] which exhibits 
different rheological characteristics from tracheal mucus [5], likely because of variations in their constitutive mucins and matrix organization [4]. Furthermore, study protocols have typically involved $400 \mu \mathrm{m}$ to several $\mathrm{mm}$ thick mucus layers exposed to relatively large volumes of drug preparations to reproduce conditions in the gastro-intestinal tract [6,7]. Such experimental designs are not pertinent to aerosolized drugs which, in the clinic, directly land onto the airway mucosa.

Considering the mucus layer is the first biological component encountered by inhaled drugs in the upper airways, it is surprising that very few studies so far have evaluated the impact of airway mucus on drug absorption in the lungs. In the 1990s, the permeability of the model antibiotics p-aminosalicylic acid, isoniazid, and pyrazinamide was compared through a buffer solution, cystic fibrosis mucus, and pig gastric mucus [8]. Mucus secretions retarded the diffusion of all three drugs with the disease model having the greatest impact due to its higher viscosity. However, in that early work, the donor compartment of the diffusion system contained a non-physiologic large volume of drug solution. Two decades later, the permeation of gentamicin sulfate [9] and ketoprofen lysinate [10] formulated as inhalation dry powders was reported to be significantly delayed in modified Franz cells when these incorporated a layer of artificial cystic fibrosis mucus or cystic fibrosis sputum, respectively. A common limitation of the three aforementioned studies is that the thickness of the mucus layers $(\sim 3 \mathrm{~mm})$ was not clinically representative. Recently, our group developed a more physiologically realistic in vitro model featuring $\sim 100 \mu \mathrm{m}$ thick layers of porcine tracheal mucus formed at the surface of semi-permeable Transwell ${ }^{\circledR}$ cell culture inserts [11]. Salbutamol sulfate or indomethacin inhalation powders were then sprayed onto the mucus layers using a custom-made deposition system. Interestingly, mucus hindered salbutamol transport but enhanced that of indomethacin, suggesting it promoted the dissolution of poorly water soluble drugs such as indomethacin.

Herein, we intended to apply drugs onto mucus layers as solution aerosols in order to differentiate the effect of airway mucus on drug diffusion from its role on particle solubilisation. Several sophisticated devices such as the CULTEX ${ }^{\circledR}[12]$, VITROCELL ${ }^{\circledR}[13]$, or ALICE-CLOUD [14,15] systems have been employed to directly expose cell culture models of the airway epithelium to aerosolized droplets. However, they necessitate technical expertise and generate low-dose prolonged exposures that are more suited to environmental toxicology studies than to drug transport measurements. In contrast, the MicroSprayer ${ }^{\circledR}$ IA-1C aerosolizer that was initially developed to facilitate the intratracheal administration of nebulized drugs in laboratory animals is straightforward to operate, only requires small volumes of drug formulations and delivers bolus doses alike therapeutic aerosols. Accordingly, it has successfully been used to expose cell cultures to aerosolized liquids [13,16-18]. The spray generated by the MicroSprayer ${ }^{\circledR}$ has typically been directed towards one single cell layer. Lengthy experiments were then required to obtain a satisfactory number of replicates. Furthermore, in such configuration, cells might be damaged by the power of the spray and exposed to doses that are much higher than in a clinical scenario.

The overarching aim of this study was to develop a simple in vitro system to investigate the parameters affecting compound interactions with airway mucus in physiologically relevant conditions. We first evaluated the retardation effect of layers of porcine tracheal mucus mounted on Transwell ${ }^{\circledR}$ inserts on the permeation of a range of fluorescent dyes with various physico-chemical properties. We then optimized a deposition system based on the MicroSprayer ${ }^{\circledR}$ aerosolizer to simultaneously and reproducibly expose multiple biological layers to solution aerosols and applied this to determine the impact of airway mucus on the diffusion of inhaled bronchodilators. The compounds tested exhibited various permeation profiles across the mucus layers with hydrophobicity appearing as the predominant but not the sole factor driving their affinity towards airway secretions. 


\section{Materials and Methods}

\subsection{Materials}

Transwell ${ }^{\circledR}$ inserts $(1.2 \mathrm{~mm}$ diameter) and well plates were purchased from Corning Ltd (Loughborough, UK). Indacaterol maleate was purchased from MedChemExpress (MCE ${ }^{\circledR}$, Monmouth Junction, NJ, USA). Formic acid and HPLC grade solvents for sample analysis were purchased from Fisher Scientific (Loughborough, UK). HPLC grade water was produced with a Purelab Ultra ELGA water purification system (Veolia Water Solutions and Technologies, Birmingham, UK). Unless specified, all other chemicals and reagents were obtained from Sigma-Aldrich (Poole, UK).

\subsection{Coating of Transwell ${ }^{\circledR}$ Inserts with Porcine Tracheal Mucus}

Mucus was collected from the trachea of healthy adult pigs slaughtered at a local abattoir, cleared of blood contaminants and stored at $-20^{\circ} \mathrm{C}$ as previously described [11]. Freezing has been demonstrated to affect neither the viscoelastic properties of native mucus nor drug coefficient of diffusion across mucus models [19]. After thawing, $12 \mu \mathrm{L}$ of mucus were resuspended in $0.1 \mathrm{M} \mathrm{NaCl}$ to reach a final volume of $300 \mu \mathrm{L}$. Twelve microliters were previously found to be the lowest volume of mucus providing full coverage of the Transwell ${ }^{\circledR}$ membrane [11]. The mucus suspension obtained was pipetted into Transwell ${ }^{\circledR}$ inserts housed in 12-well cell culture plates. The plates were centrifuged at $1500 \mathrm{rpm}$ for $15 \mathrm{~min}$; the supernatant were removed and $500 \mu \mathrm{L}$ of Hank's Balanced Salt Solution (HBSS) were placed into the wells. The plates were stored overnight to allow the mucus to stabilize and excess water to evaporate.

\subsection{Permeation of Fluorescent Dyes across Mucus Layers}

Mucus layers were formed at the surface of $0.4 \mu \mathrm{m}$ pore size polyester Transwell ${ }^{\circledR}$ membranes as described above. Solutions $(100 \mu \mathrm{M})$ of the fluorescent dyes Lucifer yellow $\mathrm{CH}$ dipotassium, Rhodamine B, Rhodamine 123, and Rose Bengal were prepared in HBSS while fluorescein isothiocyanate (FITC, $10 \mu \mathrm{M}$ ) was dissolved in HBSS containing $0.1 \%$ dimethyl sulfoxide (DMSO). Fifty $\mu \mathrm{L}$ of each dye solution pre-warmed to $37^{\circ} \mathrm{C}$ were added to the apical chamber of bare inserts or inserts supporting a mucus layer and $500 \mu \mathrm{L}$ of fresh pre-warmed HBSS (or HBSS + 0.1\% DMSO in experiments with FITC) were added to the basolateral chamber. Samples $(200 \mu \mathrm{L})$ were withdrawn from the basolateral side at pre-determined time points and replaced with $200 \mu \mathrm{L}$ of fresh pre-warmed HBSS/HBSS $+0.1 \%$ DMSO. The plate containing the Transwell ${ }^{\circledR}$ inserts was maintained at $37{ }^{\circ} \mathrm{C}$ on an orbital shaker (60 rpm) between sampling times. One hundred $\mu \mathrm{L}$ of each withdrawn sample were transferred to a black 96-well plate (Nunc F96, Scientific Laboratory Supplies, Nottingham, UK) for fluorescence measurements using a Tecan (SPARK 10M) plate-reader (Lucifer yellow: $\lambda_{\mathrm{ex}}=427 \mathrm{~nm}$ and $\lambda_{\mathrm{em}}=535$ $\mathrm{nm}$; FITC: $\lambda_{\mathrm{ex}}=490 \mathrm{~nm}$ and $\lambda_{\mathrm{em}}=540 \mathrm{~nm}$; Rhodamine B: $\lambda_{\mathrm{ex}}=544 \mathrm{~nm}$ and $\lambda_{\mathrm{em}}=680 \mathrm{~nm}$; Rhodamine 123: $\lambda_{\mathrm{ex}}=540 \mathrm{~nm}$ and $\lambda_{\mathrm{em}}=590 \mathrm{~nm}$; Rose Bengal: $\lambda_{\mathrm{ex}}=549 \mathrm{~nm}$ and $\left.\lambda_{\mathrm{em}}=620 \mathrm{~nm}\right)$. The dye concentration in the samples was determined from calibration curves prepared in HBSS or HBSS $/ 0.1 \%$ DMSO (FITC). Experiments were performed across four mucus layers or empty Transwell ${ }^{\circledR}$ inserts $(\mathrm{n}=4)$. In addition, FITC transport was assessed using four different batches of mucus $(\mathrm{N}=4 ; \mathrm{n}=4)$.

\subsection{Optimization of the Aerosolization System}

In order to deposit aerosolized drug solutions at the surface of the mucus layers, a MicroSprayer ${ }^{\circledR}$ Aerosoliser Model IA-1C (Penn-Century. Inc. Wyndmoor, PA, USA) was mounted in a vacuum glass desiccator featuring an internal deposition surface (diameter: $18.4 \mathrm{~cm}$ ) positioned $20.0 \mathrm{~cm}$ below the port entrance (Figure 1). The air-free atomizer of the MicroSprayer ${ }^{\circledR}$ was secured inside a stopper and held in a vertical position facing the centre of the internal surface thanks to a custom-made external stand. Dose volume "spacers" ( 25 or $50 \mu \mathrm{L}$ ) were attached to the plunger of the high-pressure syringe 
to facilitate precise delivery of defined volumes of aerosolised solutions. To ensure the production of a uniform aerosol spray, the plunger was actuated in a sharp and firm motion.

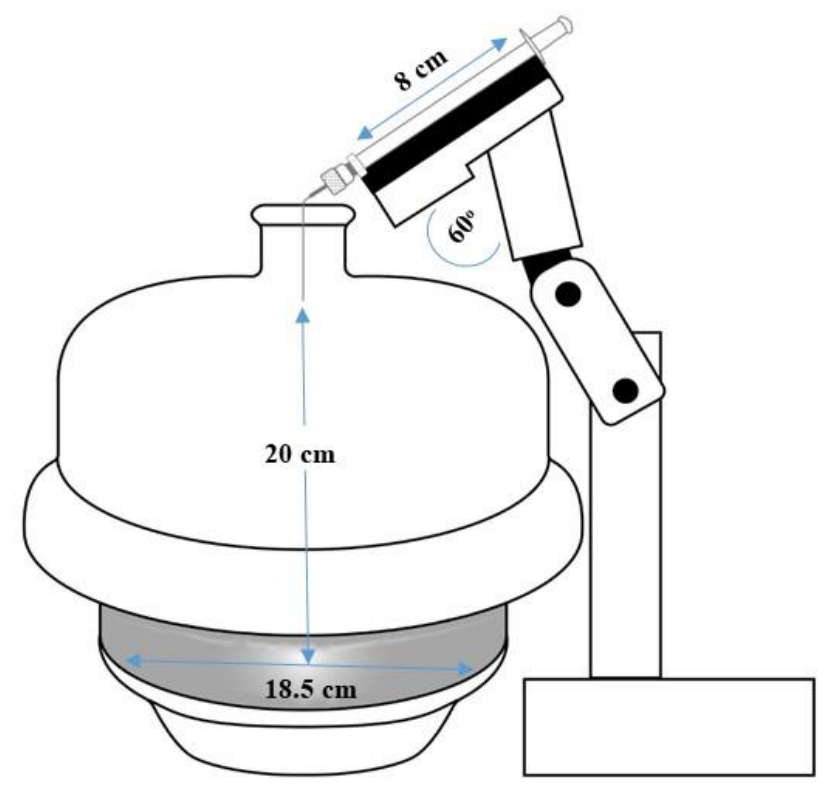

Figure 1. Schematic representation of the deposition system.

The semi-permeable membrane of four Transwell ${ }^{\circledR}$ inserts was covered with clean glass coverslips (1.2 cm in diameter, VWR, Lutterworth, UK) and those were placed inside Petri dish lids. The inserts were arranged on the internal glass desiccator surface to occupy the four corners $(A, B, C$, and D) of a virtual square centered below the tip of the MicroSprayer ${ }^{\circledR}$ and covering a surface of 20,50, or $100 \mathrm{~cm}^{2}$. A solution of Lucifer yellow (1 mM in HBSS) was loaded into the Penn-Century high-pressure syringe and various volumes $(50,100$, and $200 \mu \mathrm{L})$ were then sprayed inside the desiccator chamber. One hundred $\mu \mathrm{L}$ of HBSS were added to each Transwell ${ }^{\circledR}, 70 \mu \mathrm{L}$ were collected back from each insert and transferred to a black 96 well plate for fluorescence reading. Experiments were performed in triplicate for each volume sprayed and geometrical arrangement $(\mathrm{N}=3)$.

\subsection{Permeation across Mucus Following Aerosol Deposition}

The transport of Lucifer yellow and various bronchodilators across mucus layers spread at the surface of 0.4 or $3.0 \mu \mathrm{m}$ pore size polyester Transwell ${ }^{\circledR}$ membrane was assessed and compared to their diffusion in corresponding empty inserts.

The MicroSprayer ${ }^{\circledR}$ was loaded with Lucifer yellow $(1 \mathrm{mM})$, ipratropium bromide, glycopyrronium bromide, salbutamol sulphate (all three drugs at a final concentration of $10 \mathrm{mM}$ ) dissolved in HBSS or formoterol and indacaterol maleate solutions $(1 \mathrm{mM})$ prepared in HBSS with $10 \%$ of DMSO. A volume of $200 \mu \mathrm{L}$ was sprayed onto mucus covered or empty Transwell ${ }^{\circledR}$ inserts displayed according to the $50 \mathrm{~cm}^{2}$ geometrical arrangement described above. The inserts were immediately transferred to a 12-well plate and each well was filled with $500 \mu \mathrm{L}$ of HBSS $\pm 10 \%$ DMSO depending on the test compound. The plate was incubated at $37^{\circ} \mathrm{C}$ on an orbital shaker and samples $(50 \mu \mathrm{L})$ were withdrawn from the basolateral chambers after pre-determined time points. Each sample was replaced with an equivalent volume of HBSS ( $\pm 10 \%$ DMSO).

Following the last sampling time point, the apical chamber was washed using $200 \mu \mathrm{L}$ of HBSS $( \pm 10 \%$ DMSO) for quantification of the residual non-permeated test compound. The washed mucus layers were collected, vortexed for one minute, then centrifuged at 14,000 rpm for 5 min and the supernatants were sampled for drug content analysis. The amount of test compound detected in the wash samples was added to the cumulative amount recovered in the basolateral chamber to estimate 
the dose deposited onto the mucus layers or semi-permeable membranes upon aerosolisation of the test solutions.

Lucifer yellow concentrations were determined through fluorescence measurements while drug samples were analyzed by LC-MS/MS.

Experiments were performed in triplicate (Lucifer yellow) or quadruplicate (bronchodilators) using four Transwell ${ }^{\circledR}$ inserts per replicate $(n=4)$.

\subsection{Drug Sample Analysis}

Samples obtained from the drug permeation experiments were analyzed by LC-MS/MS.

The LC system comprised an Agilent Hewlett Packard series 1100 coupled with a Micromass Quattro Ultima Pt mass spectrometer (Waters, Milford, MA, USA) equipped with an electrospray ion source operated in positive mode. An ACE3 C18 $(3 \mu \mathrm{m}, 150 \mathrm{~mm} \times$ i.d. $2.1 \mathrm{~mm})$ column fitted with a C18 guard cartridge was used for all analysis.

Ipratropium bromide, glycopyrronium bromide, formoterol, and indacaterol maleate were processed similarly. All samples were diluted 1:3 with cold methanol containing $5 \mathrm{nM}$ of the internal standard (glycopyrronium bromide, ipratropium bromide, indacaterol maleate, and formoterol, respectively) before being stored overnight at $-20^{\circ} \mathrm{C}$. The samples were vortexed for one minute and centrifuged at $5000 \mathrm{rpm}$ for five minutes at $4{ }^{\circ} \mathrm{C}$. The supernatants were diluted (1:1) with $0.1 \%$ formic acid in water, transferred to LC-MS vials and stored in a refrigerator until analysis.

On the day of analysis, a $10 \mu \mathrm{L}$ volume was injected into the LC-MS system. Samples were run at $0.2 \mathrm{~mL} / \mathrm{min}$ applying a phase gradient, where phase A consisted of MilliQ water containing $0.1 \%$ formic acid and phase B consisted of methanol containing $0.1 \%$ formic acid. The gradient started at $45 \%$ of phase $B$, then increased to $90 \%$ over two minutes before returning to $45 \%$ over $3.5 \mathrm{~min}$ and being maintained at that level until the end of the $8.5 \mathrm{~min}$ run time. A source temperature of $125^{\circ} \mathrm{C}$, a desolvation temperature of $350^{\circ} \mathrm{C}$ and a collision energy of $28 \mathrm{kV}$ (ipratropium and glycopyrronium), $18 \mathrm{kV}$ (formoterol) or $30 \mathrm{kV}$ (indacaterol) were applied.

Salbutamol sulphate samples were diluted 1:1 with methanol, vortexed for a minute and centrifuged at $5000 \mathrm{rpm}$ for five minutes at $4{ }^{\circ} \mathrm{C}$. The supernatant were diluted 1:1 with phase A which consisted of an aqueous solution containing $0.1 \% v / v$ formic acid and ammonium formate $20 \mathrm{mM}$ (pH 3.8). $50 \mu \mathrm{L}$ of the resulting solution was injected into the LC-MS/MS system for quantification. Samples were run at $0.2 \mathrm{~mL} \mathrm{~min}^{-1}$ isocratically using a 50:50 mixture of phase A and methanol as the mobile phase. The source temperature was set at $125^{\circ} \mathrm{C}$, the desolvation temperature was fixed at $350{ }^{\circ} \mathrm{C}$ and the collision energy applied was $20 \mathrm{kV}$.

\subsection{Statistical Analysis}

Statistical analysis was performed using GraphPad Prism 6.02. Unpaired $t$-tests (multiple comparisons) and ANOVA two-way analysis (with Tukey's multiple comparison tests) were used to compare data between two groups or more than two groups, respectively. Differences between experimental groups were considered significant when a $p$-value lower than 0.05 was obtained.

\section{Results and Discussion}

Due to the complexity of the lung anatomy, bio-relevant in vitro respiratory models are necessary to gain a deeper understanding of the parameters influencing the disposition of inhaled drugs following their deposition onto the airway mucosa. Mucus-drug interactions are particularly difficult to investigate in vivo, which incentivizes us to develop a model of airway mucus based on thin layers of native porcine tracheal mucus coating the semi-permeable membrane of Transwell ${ }^{\circledR}$ inserts [11]. Pig mucus was used as it is similar to human mucus [20] and more readily accessible. In our previous study, we showed that our pig tracheal mucus samples exhibited similar rheological properties and internal structure as those reported for human airway secretions [11]. 
Porcine tracheal mucus has previously been used to assess the muco-penetrative properties of drug nanocarriers designed for the inhaled route [21]. On the other hand, its effect on the permeation of small molecules has not been investigated, at the exception of our recent study on inhalation dry powders [11]. In the present work, we assessed the role of compound physico-chemical characteristics on their permeation through porcine tracheal mucus layers, adapted our previously described deposition system [11] for production of liquid aerosols and exploited this to study the interactions of inhaled bronchodilators with airway secretions.

\subsection{Impact of Airway Mucus on the Permeation of Fluorescent Dyes}

We recently reported that mucus delayed the transport of the positively charged water soluble bronchodilator salbutamol but, in contrast, facilitated that of the negatively charged poorly soluble anti-inflammatory agent indomethacin after both drugs were exposed to the mucus layers as solid particle sprays using a bespoke deposition system [11]. As earlier studies with gastro-intestinal mucus had reported stronger binding for lipophilic molecules [3], our indomethacin data were not anticipated. They were nevertheless attributed to solubilisation enhancement properties of mucus and/or charge repulsion between the drug and the negatively charged mucin fibers. It is nevertheless possible that factors promoting compound interactions with mucus are dependent on the regional origin of the secretions. Mucus from different parts of the body are indeed well known to vary in mucin structure and scaffold organization [4].

Therefore, we measured the diffusion of diverse fluorescent dyes encompassing a broad range of physico-chemical properties across our mucus layers or empty Transwell ${ }^{\circledR}$ inserts in an attempt to uncover the molecular characteristics that might promote binding to airway mucus. Five dyes were selected for the study: Rhodamine B, a polar $(\log P:-1.1)$ amphoteric molecule; Rhodamine 123, a relatively hydrophilic ( $\log \mathrm{P}: 1.5)$ weak base that is partially ionized at $\mathrm{pH} 7.4$ and three negatively charged dyes of increasing hydrophobicity; i.e., Lucifer yellow (logP: 2.6); FITC (logP: 4.5) and Rose Bengal $(\log P>6)$. In this initial set of experiments, dye solutions were pipetted onto the layers to reflect protocols applied in related studies with gastro-intestinal mucus. Due to its poor solubility in water, DMSO was required to prepare solutions of FITC. However, the solvent did not to affect transport across the mucus layers when applied at a concentration up to 10\% (Supplementary Materials, Figure S1). The reproducibility of the mucus samples was assessed by measuring FITC permeability across layers prepared from four different batches of mucus. The four separate permeation profiles of the dye were identical (Figure 2D, $p>0.05$ ), demonstrating the reliability of the model.

The presence of a mucus layers at the surface of the Transwell ${ }^{\circledR}$ inserts had distinct effects on the diffusion of the dyes into the basolateral chamber over time. Mucus had minimal impact on the permeation of Rhodamine B with only a slight retardation effect detected over the first $30 \mathrm{~min}$ of transport (Figure $2 \mathrm{~A}, p<0.05$ ) and over $95 \%$ of the dose applied recovered in all receiver compartments after 120 min (Figure 2A, $p>0.05$ ). The permeation profiles of Rhodamine 123 with/without mucus were identical over the first $40 \mathrm{~min}$ (Figure $2 \mathrm{~B}, p>0.05$ ). Thereafter, small but significant differences emerged (Figure 2B, $p<0.05$ ) and by the end of the experiment, $90 \pm 4(\mathrm{SD}) \%$ of the dye had permeated the mucus vs $99 \pm 1$ (SD)\% for bare inserts $(p<0.05)$. In the case of Lucifer yellow, a $\sim 10 \%$ lower diffusion across mucus was observed over its entire permeation profile (Figure $2 C, p<0.05$ ), which resulted in $88 \pm 7$ (SD)\% of the dose transported within $2 \mathrm{~h}$ as compared to $98 \pm 2(\mathrm{SD}) \%$ across empty inserts. FITC transport was more dramatically impaired by mucus as only $36 \pm 4$ (SEM) $\%$ of the initial dose permeated through the layers in 120 min while $92 \pm 2$ (SEM)\% was able to cross the semi-permeable membrane of the Transwell ${ }^{\circledR}$ over the same period of time (Figure $2 \mathrm{D}, p<0.05$ at all sampling points). Similarly, mucus markedly delayed the diffusion of Rose Bengal in the receiver chamber, reducing its permeation over $2 \mathrm{~h}$ from $99 \pm 3$ (SD)\% to $43.7 \pm 0.7$ (SD)\% of the donor dose (Figure $2 \mathrm{E}, p<0.05$ at all sampling points). 

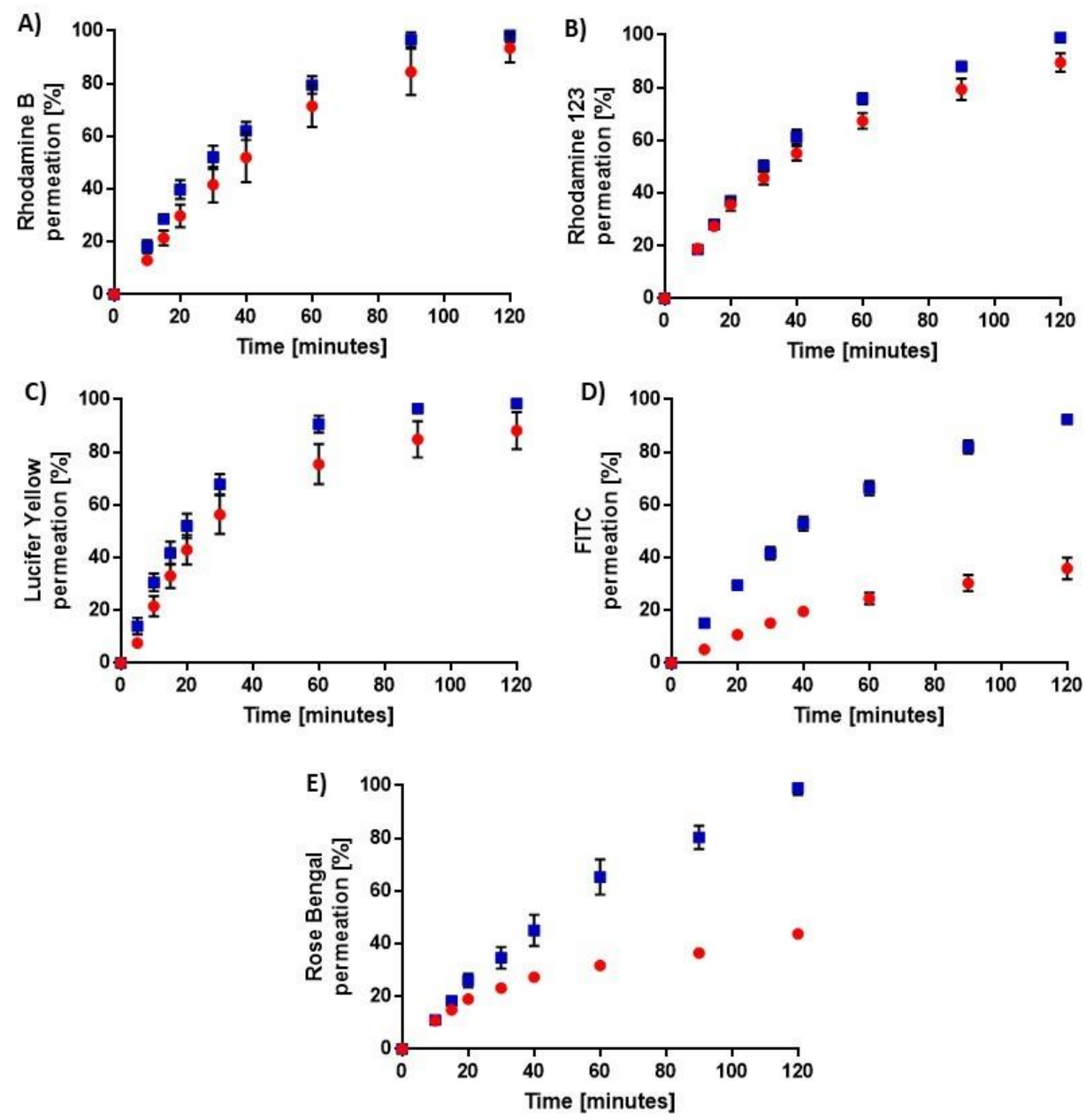

- Mucus layer - Empty Transwell

Figure 2. Permeation profiles of fluorescent dyes across mucus layers spread at the surface of $0.4 \mu \mathrm{m}$ pore size polyester Transwell ${ }^{\circledR}$ inserts and corresponding empty inserts. $50 \mu \mathrm{L}$ of the dye solutions were added to the apical chambers at $\mathrm{t}=0$. A: Rhodamine B; B: Rhodamine 123; C: Lucifer yellow; $\mathbf{D}$ : FITC; E: Rose Bengal. Data are expressed as cumulative percentage of the initial donor dose recovered in the basolateral compartment as a function of time. They are presented as mean \pm SD $(n=4)$ or mean \pm SEM (FITC; $\mathrm{N}=4, \mathrm{n}=4$ ).

Despite the small number of molecules tested, hydrophobicity stood out as a key parameter driving compound interactions with airway mucus while charge had much less of an impact. This is in line with the inverse relationship previously obtained between drug diffusion coefficients in native intestinal pig mucus and their lipophilicity as well as the minor role of compound charge in mucus binding observed in that model [22]. In contrast, no correlation between transport rate and $\log \mathrm{D}$ was found in purified pig gastric mucin [22]. It was later demonstrated that hydrophobic interactions occur preferentially with the lipids contained in raw intestinal pig mucus samples rather than with mucin itself [23].

The high concentration of lipids found in intestinal mucus, e.g., 37\% in dry weight [23], is often attributed to digested food. Nevertheless, human airway mucus has also been shown to include a high lipidic fraction, i.e., $25-40 \%$ in dry weight depending on the sample [24]. Lipids were very likely present in our mucus model since only a cleaning step to remove blood contaminants was carried out during the preparation of the Transwell ${ }^{\circledR}$-supported layers. Binding to lipids was therefore the most probable mechanism underlying the marked effect of pig tracheal mucus on the permeability of FITC and Rose Bengal, the two highly hydrophobic dyes tested in this study (Figure 2). 
Our data suggests that low molecular weight compounds might interact with airway or gastro-intestinal mucus in a similar fashion. It would however be interesting to compare the permeation of the same series of molecules across both tracheal and gastro-intestinal mucus in identical diffusion systems. This would also indicate whether airway mucus offers any benefit in inhaled drug permeation studies or alternatively, could be substituted by gastro-intestinal mucus whose yield per animal is much higher than that of tracheal secretions.

\subsection{Optimization of the Aerosolization System}

In order to mimic the clinical situation, aerosolized drugs must be directly deposited onto any in vitro model of the airway mucosa. To achieve this, our previously described deposition system was modified by replacing the PennCentury ${ }^{\mathrm{TM}}$ Dry Powder Insufflator [11] with a MicroSprayer ${ }^{\circledR}$ Aerosoliser to allow the exposure of air-facing mucus layers to liquid aerosols. In order to minimize the physical impact of the liquid sprays on the mucus model, four Transwell ${ }^{\circledR}$ inserts were positioned $20 \mathrm{~cm}$ below the tip of the Aerosoliser and at variable distance from the centre of the spray in an arrangement shaping a squared area of either 20,50, or $100 \mathrm{~cm}^{2}$ (Figure 3). The extent and reproducibility of the dose delivered onto the inserts was optimized by spraying different volumes of Lucifer yellow solutions inside the aerosolization chamber.

As expected, for all geometric configurations, a spray volume of $200 \mu \mathrm{L}$ resulted in a higher average amount of fluorescent dye delivered to the inserts than a spray of 50 or $100 \mu \mathrm{L}$ (Figure 3). When the inserts were placed at the shortest distance from the center of the deposition platform, they received highly variable doses of the dye with any of the three volumes of Lucifer yellow aerosolized (coefficient of variation up to $72 \%$ ) (Figure $3 \mathrm{~A}$ ). Positioning the Transwell ${ }^{\circledR}$ inserts at the outskirts of the deposition chamber improved the reproducibly of the deposited dose and no significant difference was noted between the amount of dye reaching the four inserts when $100 \mu \mathrm{L}$ of the solution was released from the MicroSprayer ${ }^{\circledR}(p>0.05$, Figure 3B). However, that amount was very low $(<0.06 \mathrm{nmol})$ and could have led to analytical challenges during permeation studies across the mucus layers. The intermediate geometric arrangement in which the inserts delineated a $50 \mathrm{~cm}^{2}$ square provided a compromise between efficient deposition and dose reproducibility. In that set-up, spraying a volume of $200 \mu \mathrm{L}$ resulted in $0.12 \pm 0.04 \mathrm{nmol}$ (which corresponded to $\sim 120 \mathrm{~nL}$ ) of the fluorescent dye consistently landing onto the four Transwell ${ }^{\circledR}$ inserts $(p<0.05$, Figure 3C).

Applying those experimental conditions, Lucifer yellow was then sprayed onto mucus layers spread at the surface of the Transwell ${ }^{\circledR}$ inserts or into bare inserts and its diffusion into the basolateral compartment was monitored over $120 \mathrm{~min}$. The transport of the aerosolized dye across both mucus and the semi-permeable membrane alone was initially faster than after addition of $50 \mu \mathrm{L}$ of the dye solution into the donor chambers (Figure 2D). Indeed, after $10 \mathrm{~min}, 48 \% \pm 5 \%$ of the deposited aerosol dose had diffused through air-interfaced mucus layers (Figure 4$)$ as compared to $22 \% \pm 4 \%(p<0.05)$ when these were submerged by the dye solution (Figure 2D). Permeation across the bare Transwell ${ }^{\circledR}$ membrane was rapid with $>90 \%$ of the dose recovered in the receiver chamber after 20 min (Figure 4 ). This can probably be explained by the absence of a bulk solution and unstirred water layer acting as diffusion barriers upon direct exposure of the mucus or the semi-permeable membrane to the dye. However, the hindering effect of mucus on Lucifer yellow diffusion was more pronounced upon aerosolization of the dye, as indicated by the clear difference in its permeation profiles in presence or absence of airway secretions (Figure 4), unlike those across submerged mucus layers and Transwell ${ }^{\circledR}$ membranes (Figure 2D). In submerged conditions, mucus could have been diluted by the dye solution and would therefore have formed a rather lose layer at the surface of the Transwell ${ }^{\circledR}$ inserts allowing a freer diffusion of the solutes. This illustrates the importance of investigating drug interactions with biological barriers using in vitro systems that closely resemble the in vivo environment being modelled. 
A)

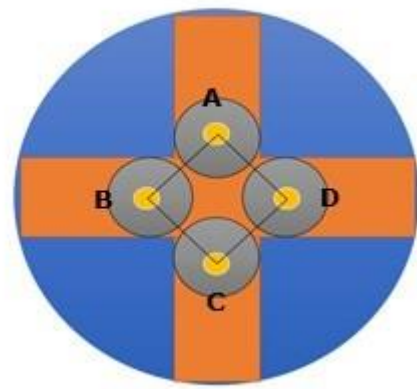

B)

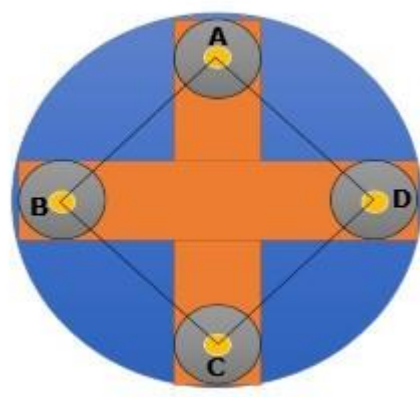

C)
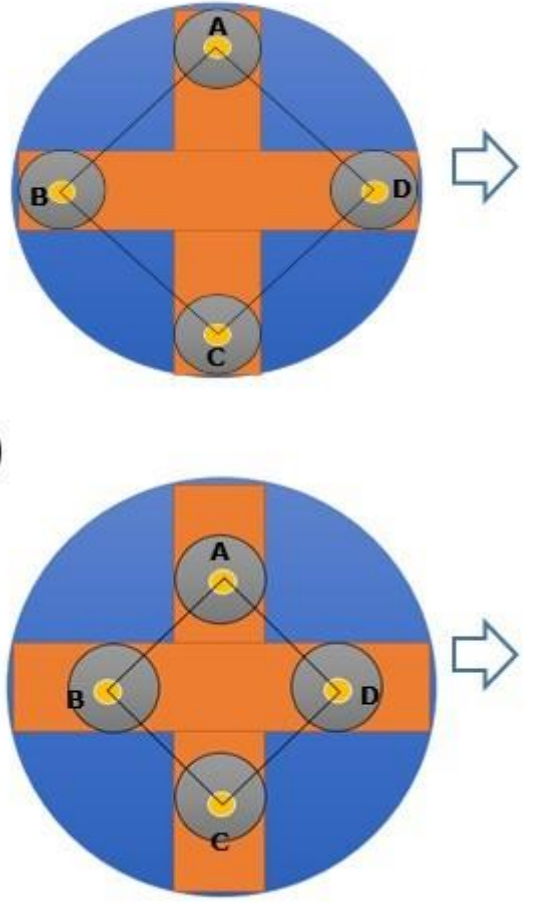

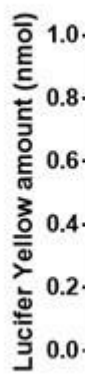

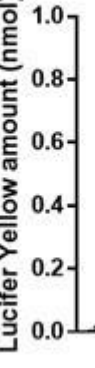

Position A

口 Position B

口 Position C

- Position D

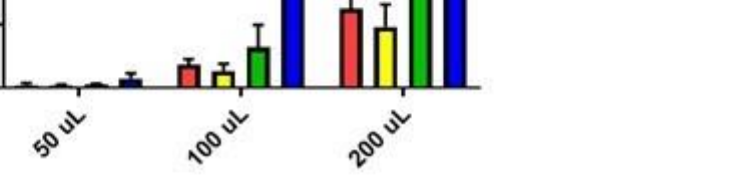

Sprayed Volume

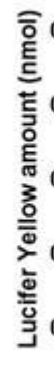
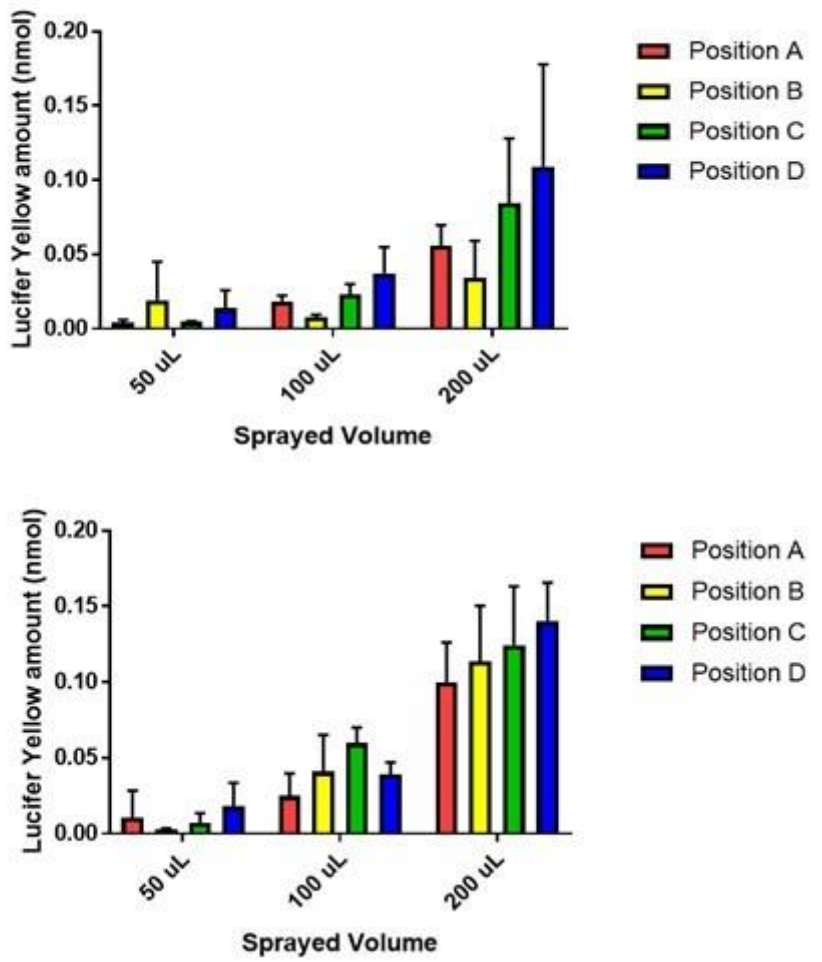

Figure 3. Doses of Lucifer yellow deposited on Transwell ${ }^{\circledR}$ inserts when sprayed from a distance of $20 \mathrm{~cm}$. Inserts were arranged to cover a $20(\mathbf{A}), 100(\mathbf{B})$, or $50(\mathbf{C}) \mathrm{cm}^{2}$ surface area. Data are presented as mean $\pm \mathrm{SD}(\mathrm{n}=3)$. 


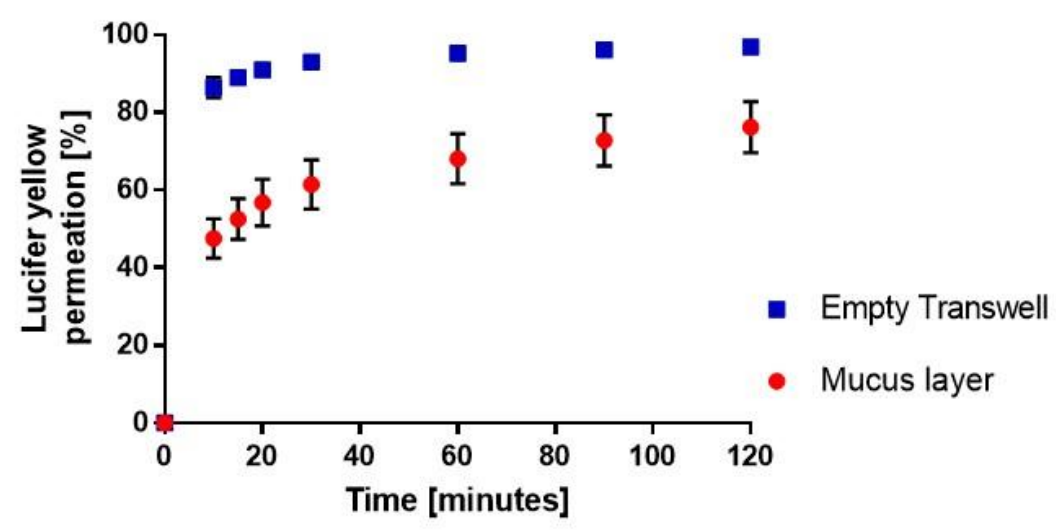

Figure 4. Permeation profiles of Lucifer yellow after it was sprayed at a distance of $20 \mathrm{~cm}$ onto mucus layers prepared in $0.4 \mu \mathrm{m}$ pore size polyester Transwell ${ }^{\circledR}$ inserts or corresponding empty inserts arranged to cover a $50 \mathrm{~cm}^{2}$ surface area. Data are expressed as cumulative percentage of the deposited dose recovered in the basolateral compartment as a function of time. They are presented as mean \pm $\operatorname{SEM}(\mathrm{N}=3, \mathrm{n}=4)$.

\subsection{Interactions of Inhaled Bronchodilators with Airway Mucus}

The deposition system was then employed to evaluate the influence of airway mucus on the pulmonary absorption of marketed inhaled bronchodilators of the M3 antagonist (ipratropium and glycopyrronium) and $\beta_{2}$-agonist classes (salbutamol, formoterol and indacaterol). Those drugs are either cationic or zwitterionic at neutral $\mathrm{pH}$ but significantly differ in their lipophilicity (Table 1).

Table 1. Properties, dose deposited and permeation rate through mucus layers of the bronchodilators tested.

\begin{tabular}{cccccccc}
\hline Compound & $\begin{array}{c}\text { Chemical } \\
\text { Class }\end{array}$ & LogP & MW & $\begin{array}{c}\text { H-Bond } \\
\text { (Donor/Acceptor) }\end{array}$ & $\begin{array}{c}\text { Dose } \\
\text { Deposited (ng) }\end{array}$ & $\begin{array}{c}\mathbf{T 5 0}^{\mathbf{a}} \\
(\mathbf{m i n})\end{array}$ & $\begin{array}{c}\mathbf{T}^{\mathbf{2}} \mathbf{b}^{\mathbf{b}} \\
(\mathbf{m i n})\end{array}$ \\
\hline Ipratropium & Quaternary & -1.8 & 412 & $1 / 4$ & $442 \pm 76$ & $7.0 \pm 0.5$ & $<5$ \\
Glycopyronnium & Quaternary & -1.4 & 398 & $1 / 4$ & $384 \pm 92$ & $5.4 \pm 0.9$ & ND \\
Salbutamol & Base & 1.4 & 577 & $10 / 12$ & $687 \pm 116$ & $19 \pm 3$ & $12.4 \pm 0.8$ \\
Formoterol & Base & 2.2 & 344 & $4 / 5$ & $216 \pm 46$ & $13 \pm 3$ & $7 \pm 1$ \\
Indacaterol & Zwitterion & 4.05 & 509 & $6 / 8$ & $232 \pm 61$ & $67 \pm 11$ & $20 \pm 1$ \\
\hline
\end{tabular}

* extracted from PubChem (https://pubchem.ncbi.nlm.nih.gov); MW: molecular weight; $\mathrm{T}_{50}$ : time taken for $50 \%$ of the deposited dose to cross mucus layers mounted on ${ }^{\mathrm{a}} 0.4 \mu \mathrm{m}$ or ${ }^{\mathrm{b}} 3.0 \mu \mathrm{m}$ pore size inserts; ND: not determined; Data are presented as mean \pm SEM $(N=4, n=4)$.

The amounts deposited onto the mucus layers ranged from 200 to $700 \mathrm{ng}$ and was more dependent on the drug itself than on the concentration of the solution loaded into the MicroSprayer ${ }^{\circledR}$ (Table 1). Those variations might be related to changes in the viscosity of the test solutions and the geometry of the spray with the investigated compounds. In a clinical scenario, if one considers $20-50 \%$ of the dose emitted from the inhaler reach the lungs [25], a typical patchy drug distribution [25] and the surface area of the upper airways, the deposited dose per surface area is likely to be even lower. However, replicating such doses in vitro is not feasible as they would result in undetectable concentrations of permeated drugs.

Ipratropium and glycopyrronium exhibited indistinguishable permeation profiles across the mucus layers with, respectively, $44 \% \pm 4 \%$ and $48 \% \pm 3 \%$ of the applied dose detected in the receiver chambers after the first five minutes and a plateau above $95 \%$ observed from 45 min onwards (Figure $5 \mathrm{~A}, \mathrm{~B}, p>0.05$ ). The permeation profile of both drugs then coincided with that across the empty Transwell ${ }^{\circledR}$ inserts (Figure $5 \mathrm{~A}, \mathrm{~B}, p>0.05$ ). Those data were not surprising since the two M3 antagonists share similar physicochemical properties such as a low $\log P$ value and the presence of a quaternary ammonium in their chemical structure (Table 1). 
A)

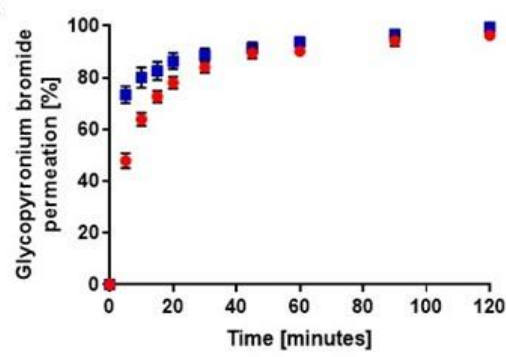

C)

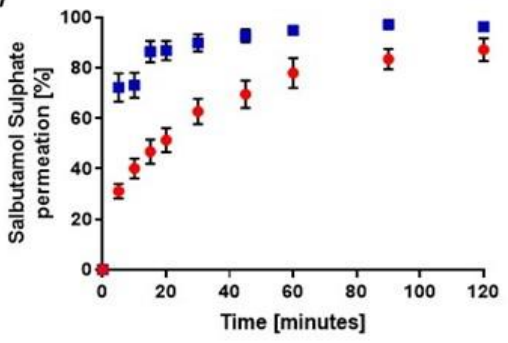

B)

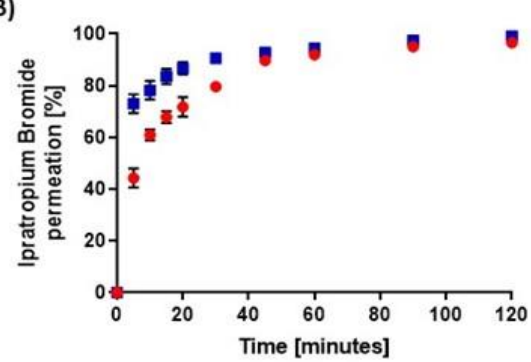

D)

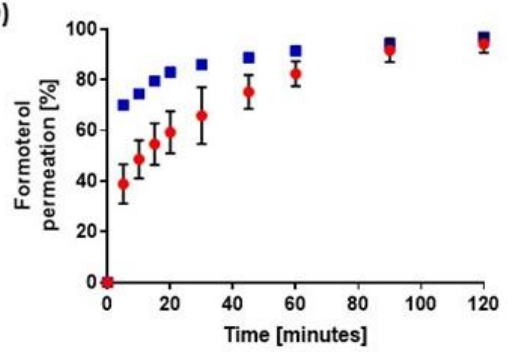

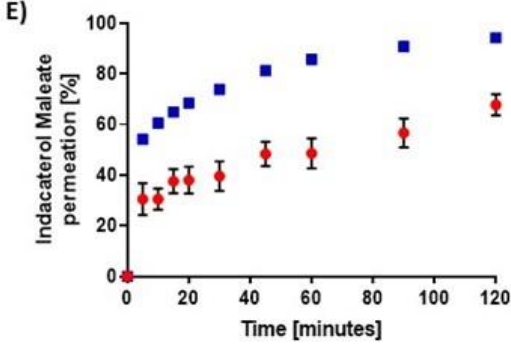

- Mucus layer a Empty Transwell

Figure 5. Permeation profiles of inhaled bronchodilators after they were sprayed at a distance of $20 \mathrm{~cm}$ onto mucus layers mounted onto $0.4 \mu \mathrm{m}$ pore size polyester Transwell ${ }^{\circledR}$ inserts or corresponding empty inserts arranged to cover a $50 \mathrm{~cm}^{2}$ surface area. A: glycopyrronium bromide; B: ipratropium bromide; C: salbutamol sulphate; D: formoterol; E: indacaterol maleate. Data are expressed as cumulative percentage of the deposited dose recovered in the basolateral compartment as a function of time. They are presented as mean $\pm \operatorname{SEM}(\mathrm{N}=4, \mathrm{n}=4)$.

In contrast, the $\beta_{2}$-agonists interacted differently with airway mucus. The diffusion of the short acting salbutamol was significantly lower across airway mucus than Transwell ${ }^{\circledR}$ inserts over all time points (Figure $5 \mathrm{C}, p<0.05$ ) and only $31 \pm 3 \%$ of the initial dose had permeated the mucus layers within the first five minutes, confirming our previous observations that mucus impairs the pulmonary absorption of that inhaled drug [11]. The transport across airway secretions of the long-acting formoterol was faster than that of salbutamol. A higher percentage of the former drug, i.e., $39 \pm 8 \%$ was recovered basolaterally five minutes post aerosolization and during the second hour of the study, the fractions that had diffused through the mucus or the bare inserts were not statistically different (Figure 5D, $p>0.05$ ). Finally, a slow permeation across mucus was evident for the ultra-long-acting indacaterol (Figure 5E). While a similar amount of the drug as for salbutamol had crossed the layers within the first $5 \mathrm{~min}$; i.e., $31 \pm 6 \%$, only two thirds of the applied dose of indacaterol was able to overcome the mucus barrier in 120 min (Figure 5E).

In line with previous observations with the different fluorescent dyes, the highly hydrophobic bronchodilator indacaterol was strongly retained within the mucus layers despite its net neutral charge while the highly hydrophilic positively charged M3 antagonists showed the weakest interactions with mucus. Hydrophobicity can nevertheless not be accounted for the relatively slow permeation of salbutamol across airway mucus, suggesting additional drug properties promote mucus binding, such as for instance, their ability to form hydrogen bonds with mucus components. Salbutamol indeed 
features a very high number of hydrogen bond acceptor and donor sites as compared to the other inhaled drugs included in this study (Table 1), which could explain its interactions with mucus were stronger than expected based on its hydrophilicity.

However, as a matter of concern, it was noticeable that the Transwell ${ }^{\circledR}$ membrane itself acted as a diffusion barrier to all five drugs but particularly to indacaterol (Figure 5). A similar rate-limiting diffusion across Transwell ${ }^{\circledR}$ inserts had previously been reported for the poorly water soluble inhaled corticosteroid ciclesonide as part of a drug dissolution study [26]. This was then corrected by replacing the $0.4 \mu \mathrm{m}$ pore size polyester membranes of the Transwell ${ }^{\circledR}$ by glass microfiber or paper filters affixed onto the plastic walls of the inserts using mild heat [26]. As a more convenient alternative, herein, we replicated ipratropium, salbutamol, formoterol and indacaterol permeation studies with mucus layers mounted onto $3.0 \mu \mathrm{m}$ instead of $0.4 \mu \mathrm{m}$ pore size polyester Transwell ${ }^{\circledR}$. Using those more porous inserts, at least $85 \%$ of the dose deposited onto the membrane was detected in the receiver chambers within the first $5 \mathrm{~min}$ for all four drugs tested (Figure 6).
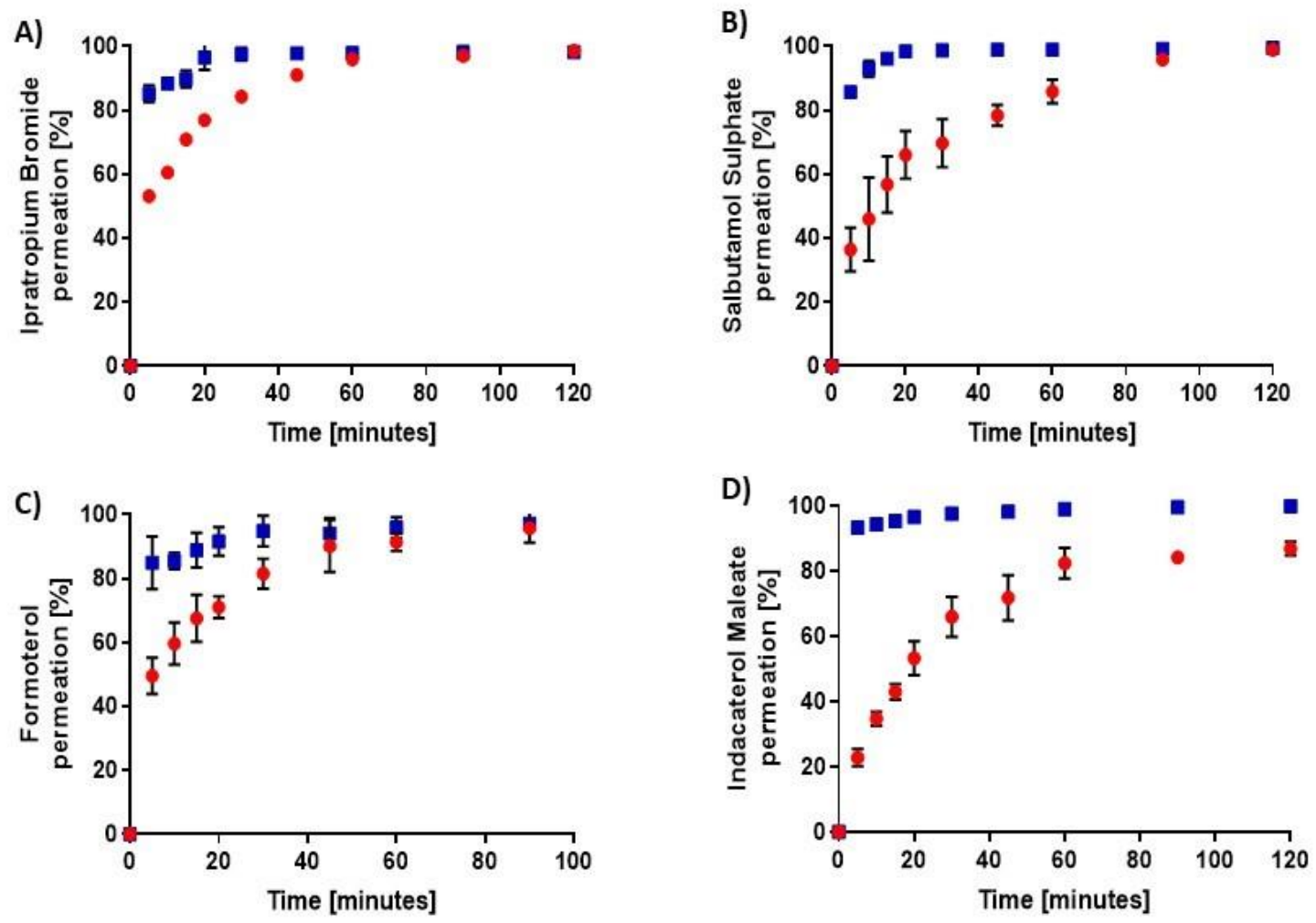

\section{- Mucus layer - Empty Transwell}

Figure 6. Permeation profiles of inhaled bronchodilators after they were sprayed at a distance of $20 \mathrm{~cm}$ onto mucus layers mounted onto $3.0 \mu \mathrm{m}$ pore size polyester Transwell ${ }^{\circledR}$ inserts or corresponding empty inserts geometrically arranged to cover a $50 \mathrm{~cm}^{2}$ surface area. A: ipratropium bromide; B: salbutamol sulphate; C: formoterol; D: indacaterol maleate. Data are expressed as cumulative percentage of the deposited dose recovered in the basolateral compartment as a function of time. They are presented as mean $\pm \operatorname{SEM}(\mathrm{N}=4, \mathrm{n}=4)$.

Transport across airway secretions was also less restricted than when the layers were supported by semi-permeable membranes with narrower pores, as reflected by a reduction in the time needed for $50 \%$ of the applied dose to diffuse into the basolateral compartments $\left(T_{50}\right.$, Table 1$)$. The extent of increase in transport rate was however drug dependent. The difference in $\mathrm{T}_{50}$ was negligible for ipratropium but pronounced for indacaterol (Table 1), suggesting that molecular diffusion through the Transwell ${ }^{\circledR}$ membrane can be delayed due to hydrophobic interactions. Nevertheless, the four bronchodilators ranked in the same order in terms of diffusion rate across mucus in the two types of 
inserts; i.e., ipratropium $>$ formoterol $>$ salbutamol $>$ indacaterol (Table 1), which indicates that mucus had overall a more significant impact on their permeation than the semi-permeable membrane. It is noteworthy that, in contrast to the pore size, the membrane material had a very limited influence on drug transport. Indeed, the permeation profiles of indacaterol with or without mucus in polycarbonate vs polyester Transwell ${ }^{\circledR}$ were comparable, although the drug was marginally more hindered in the former (Supplementary Materials, Figure S2). This is not surprising considering the two polymers exhibit close water contact angles; i.e., $84^{\circ}$ for polycarbonate [27] and $\sim 75^{\circ}$ for polyethylene terephthalate (PET, polyester) [28]. Accordingly, they are both considered as moderately hydrophilic with polycarbonate being slightly more hydrophobic.

Overall, our data suggest that airway mucus could interfere with the absorption of inhaled bronchodilators in the lungs. The impact of mucus on drug diffusion may nevertheless be overestimated in this model. Indeed, the thickness of the mucus layers $(\sim 100 \mu \mathrm{m})$ was 10 fold that of the mucus blanket in vivo as it was not experimentally possible to form thinner supported mucus layers that covered the entire surface of the Transwell ${ }^{\circledR}$ membranes [11]. Furthermore, inhaled bronchodilators all show a very low permeability across bronchial epithelial cells in vitro [11,29-31] and we previously reported salbutamol transport was restricted to a larger extent by epithelial layers than by mucus [11]. Therefore, the airway epithelium likely represents the major barrier to absorption for those drugs in the lungs. Binding to the mucus layer could nevertheless enhance the retention of relatively hydrophobic inhaled drugs in the pulmonary tissue, although this would presumably be associated with a loss of therapeutic activity.

\section{Conclusions}

A simple deposition system was assembled to deliver reproducible doses of liquid aerosols to multiple Transwell ${ }^{\circledR}$ inserts. It was used in this work to explore the impact of airway mucus on the permeation of a series of inhaled bronchodilators in physiologically relevant conditions but would also be suitable for exposing air-interfaced respiratory cell culture models to aerosolized drugs and formulations. Airway mucus was shown to delay the diffusion of all tested drugs, although to various extents. The number of compounds included in the study was too small to establish reliable correlations between physico-chemical properties and mucus affinity. Nevertheless, hydrophobicity emerged as one important factor. The involvement of additional drug characteristics was evident although defining these will require measuring the transport of larger series of compounds across airway mucus. This study indicates binding to mucus might affect the disposition and therapeutic activity of inhaled drugs in the lungs. Investigating their interactions with airway mucus is therefore worth pursuing, ideally using mucus from diseased rather than healthy lungs.

Supplementary Materials: The following are available online at http://www.mdpi.com/1999-4923/12/2/145/s1, Figure S1: Effect of increasing concentrations of DMSO on solute transport across mucus layers, Figure S2: Effect of the Transwell ${ }^{\circledR}$ membrane composition on molecular diffusion.

Author Contributions: Conceptualization, S.A. and C.B.; methodology, S.A.; software, not applicable.; validation, S.A., C.B., C.J.R., and S.S.; formal analysis, S.A. and C.B.; investigation, S.A.; resources, not applicable; data curation, S.A.; writing-original draft preparation, C.B.; writing—review and editing, S.A., C.J.R., and S.S.; visualization, S.A.; supervision, C.B., C.J.R., and S.S.; project administration, C.B., C.J.R., and S.S.; funding acquisition, S.A. All authors have read and agreed to the published version of the manuscript.

Funding: S.A.'s scholarship was funded by Prince Sattam Bin Abdulaziz University, Saudi Arabia. This research received no other external funding.

Acknowledgments: Catharine Ortori (School of Pharmacy, University of Nottingham) for her help with the analysis of the drug samples.

Conflicts of Interest: The authors declare no conflict of interest. The funder had no role in the design of the study; in the collection, analyses, or interpretation of data; in the writing of the manuscript, or in the decision to publish the results. 


\section{References}

1. Moon, C.; Smyth, H.D.C.; Watts, A.B.; Williams, R.O., 3rd. Delivery technologies for orally inhaled products: An update. AAPS PharmSciTech. 2019, 20, 117. [CrossRef] [PubMed]

2. Barnes, P.J.; Bonini, S.; Seeger, W.; Belvisi, M.G.; Ward, B.; Holmes, A. Barriers to new drug development in respiratory disease. Eur. Respir. J. 2015, 45, 1197-1207. [CrossRef] [PubMed]

3. Sigurdsson, H.H.; Kirch, J.; Lehr, C.M. Mucus as a barrier to lipophilic drugs. Int. J. Pharm. 2013, 453, 56-64. [CrossRef] [PubMed]

4. Murgia, X.; Loretz, B.; Hartwig, O.; Hittinger, M.; Lehr, C.M. The role of mucus on drug transport and its potential to affect therapeutic outcomes. Adv. Drug Deliv. Rev. 2018, 15, 82-97. [CrossRef]

5. Bokkasam, H.; Ernst, M.; Guenther, M.; Wagner, C.; Schaefer, U.F.; Lehr, C.M. Different macro- and micro-rheological properties of native porcine respiratory and intestinal mucus. Int. J. Pharm. 2016, 510, 164-167. [CrossRef]

6. Shaw, L.R.; Irwin, W.J.; Grattan, T.J.; Conway, B.R. The influence of excipients on the diffusion of ibuprofen and paracetamol in gastric mucus. Int. J. Pharm. 2005, 290, 145-154. [CrossRef]

7. Friedl, H.; Dünnhaupt, S.; Hintzen, F.; Waldner, C.; Parikh, S.; Pearson, J.P.; Wilcox, M.D.; Bernkop-Schnürch, A. Development and evaluation of a novel mucus diffusion test system approved by self-nanoemulsifying drug delivery systems. J. Pharm. Sci. 2013, 102, 4406-4413. [CrossRef]

8. Bhat, P.G.; Flanagan, D.R.; Donovan, M.D. Drug diffusion through cystic fibrotic mucus: Steady-state permeation, rheologic properties, and glycoprotein morphology. J. Pharm. Sci. 1996, 85, 624-630. [CrossRef]

9. Russo, P.; Stigliani, M.; Prota, L.; Auriemma, G.; Crescenzi, C.; Porta, A.; Aquino, R.P. Gentamicin and leucine inhalable powder: What about antipseudomonal activity and permeation through cystic fibrosis mucus? Int. J. Pharm. 2013, 440, 250-255. [CrossRef]

10. Stigliani, M.; Manniello, M.D.; Zegarra-Moran, O.; Galietta, L.; Minicucci, L.; Casciaro, R.; Garofalo, E.; Incarnato, L.; Aquino, R.P.; Del Gaudio, P.; et al. Rheological Properties of Cystic Fibrosis Bronchial Secretion and in Vitro Drug Permeation Study: The Effect of Sodium Bicarbonate. J. Aerosol. Med. Pulm. Drug Deliv. 2016, 29, 337-345. [CrossRef]

11. Cingolani, E.; Alqahtani, S.; Sadler, R.C.; Prime, D.; Stolnik, S.; Bosquillon, C. In vitro investigation on the impact of airway mucus on drug dissolution and absorption at the air-epithelium interface in the lungs. Eur. J. Pharm. Biopharm. 2019, 141, 210-220. [CrossRef] [PubMed]

12. Deschl, U.; Vogel, J.; Aufderheide, M. Development of an in vitro exposure model for investigating the biological effects of therapeutic aerosols on human cells from the respiratory tract. Exp. Toxicol. Pathol. 2011, 63, 593-598. [CrossRef] [PubMed]

13. Fröhlich, E.; Bonstingl, G.; Höfler, A.; Meindl, C.; Leitinger, G.; Pieber, T.R.; Roblegg, E. Comparison of two in vitro systems to assess cellular effects of nanoparticles-containing aerosols. Toxicol. In Vitro 2013, 27, 409-417. [CrossRef] [PubMed]

14. Brandenberger, C.; Mühlfeld, C.; Ali, Z.; Lenz, A.G.; Schmid, O.; Parak, W.J.; Gehr, P.; Rothen-Rutishauser, B. Quantitative evaluation of cellular uptake and trafficking of plain and polyethylene glycol-coated gold nanoparticles. Small 2010, 6, 1669-1678. [CrossRef] [PubMed]

15. Lenz, A.G.; Stoeger, T.; Cei, D.; Schmidmeir, M.; Semren, N.; Burgstaller, G.; Lentner, B.; Eickelberg, O.; Meiners, S.; Schmid, O. Efficient bioactive delivery of aerosolized drugs to human pulmonary epithelial cells cultured in air-liquid interface conditions. Am. J. Respir. Cell Mol. Biol. 2014, 51, 526-535. [CrossRef] [PubMed]

16. Blank, F.; Rothen-Rutishauser, B.M.; Schurch, S.; Gehr, P. An optimized in vitro model of the respiratory tract wall to study particle cell interactions. J. Aerosol. Med. 2006, 19, 392-405. [CrossRef]

17. Heuking, S.; Rothen-Rutishauser, B.; Raemy, D.O.; Gehr, P.; Borchard, G. Fate of TLR-1/TLR-2 agonist functionalised pDNA nanoparticles upon deposition at the human bronchial epithelium in vitro. J. Nanobiotechnol. 2013, 21, 29. [CrossRef]

18. Meindl, C.; Stranzinger, S.; Dzidic, N.; Salar-Behzadi, S.; Mohr, S.; Zimmer, A.; Fröhlich, E. Permeation of Therapeutic Drugs in Different Formulations across the Airway Epithelium In Vitro. PLoS ONE 2015, 10, e0135690. [CrossRef]

19. Groo, A.C.; Lagarce, F. Mucus models to evaluate nanomedicines for diffusion. Drug Discov. Today 2014, 19, 1097-1108. [CrossRef] 
20. Kararli, T.T. Comparison of the gastrointestinal anatomy, physiology, and biochemistry of humans and commonly used laboratory animals. Biopharm. Drug. Dispos. 1995, 16, 351-380. [CrossRef]

21. Murgia, X.; Pawelzyk, P.; Schaefer, U.F.; Wagner, C.; Willenbacher, N.; Lehr, C.M. Size-Limited Penetration of Nanoparticles into Porcine Respiratory Mucus after Aerosol Deposition. Biomacromolecules 2016, 17, 1536-1542. [CrossRef] [PubMed]

22. Larhed, A.W.; Artursson, P.; Gråsjö, J.; Björk, E. Diffusion of drugs in native and purified gastrointestinal mucus. J. Pharm. Sci. 1997, 86, 660-665. [CrossRef] [PubMed]

23. Larhed, A.W.; Artursson, P.; Björk, E. The influence of intestinal mucus components on the diffusion of drugs. Pharm. Res. 1998, 15, 66-71. [CrossRef] [PubMed]

24. Slayter, H.S.; Lamblin, G.; Le Treut, A.; Galabert, C.; Houdret, N.; Degand, P.; Roussel, P. Complex structure of human bronchial mucus glycoprotein. Eur. J. Biochem. 1984, 142, 209-218. [CrossRef]

25. Fröhlich, E. Biological Obstacles for Identifying In Vitro-In Vivo Correlations of Orally Inhaled Formulations. Pharmaceutics 2019, 11, 316. [CrossRef]

26. Rohrschneider, M.; Bhagwat, S.; Krampe, R.; Michler, V.; Breitkreutz, J.; Hochhaus, G. Evaluation of the Transwell System for Characterization of Dissolution Behavior of Inhalation Drugs: Effects of Membrane and Surfactant. Mol. Pharm. 2015, 12, 2618-2624. [CrossRef]

27. Jankowski, P.; Ogonczyk, D.; Kosinski, A.; Lisowski, W.; Garstecki, P. Hydrophobic modification of polycarbonate for reproducible and stable formation of biocompatible microparticles. Lab Chip 2011, 11, 748-752. [CrossRef]

28. Jasmee, S.; Omar, G.; Masripan, N.A.B.; Kamarolzaman, A.A.; Ashikin, A.S.; Che Ani, F. Hydrophobicity performance of polyethylene terephthalate (PET) and thermoplastic polyurethane (TPU) with thermal effect. Mater. Res. Express 2018, 5, 096304. [CrossRef]

29. Mukherjee, M.; Pritchard, D.I.; Bosquillon, C. Evaluation of air-interfaced Calu-3 cell layers for investigation of inhaled drug interactions with organic cation transporters in vitro. Int J Pharm 2012, 426, 7-14. [CrossRef]

30. Mukherjee, M.; Cingolani, E.; Pritchard, D.I.; Bosquillon, C. Enhanced expression of Organic Cation Transporters in bronchial epithelial cell layers following insults associated with asthma-Impact on salbutamol transport. Eur. J. Pharm. Sci. 2017, 106, 62-70. [CrossRef]

31. Panduga, V.; Stocks, M.J.; Bosquillon, C. Ipratropium is 'luminally recycled' by an inter-play between apical uptake and efflux transporters in Calu-3 bronchial epithelial cell layers. Int. J. Pharm. 2017, 532, 328-336. [CrossRef] [PubMed]

(C) 2020 by the authors. Licensee MDPI, Basel, Switzerland. This article is an open access article distributed under the terms and conditions of the Creative Commons Attribution (CC BY) license (http://creativecommons.org/licenses/by/4.0/). 